\title{
Verifying Visual Properties in Sentence Verification Facilitates Picture Recognition Memory
}

\author{
Diane Pecher, Kiki Zanolie, and René Zeelenberg
}

Erasmus University Rotterdam, The Netherlands

\begin{abstract}
According to the perceptual symbols theory (Barsalou, 1999), sensorimotor simulations underlie the representation of concepts. We investigated whether recognition memory for pictures of concepts was facilitated by earlier representation of visual properties of those concepts. During study, concept names (e.g., apple) were presented in a property verification task with a visual property (e.g., shiny) or with a nonvisual property (e.g., tart). Delayed picture recognition memory was better if the concept name had been presented with a visual property than if it had been presented with a nonvisual property. These results indicate that modality-specific simulations are used for concept representation.
\end{abstract}

Keywords: embodied cognition, concepts, recognition memory, representation

How do people represent the world in their memory? Recently a number of theories have proposed that people use sensorimotor processes for representing concepts and events (Barsalou, 1999; Glenberg, 1997; Goldstone \& Barsalou, 1998; Pulvermüller, 1999; Wilson, 2002; Zwaan, 2004). These theories propose that representations have many similarities to actual experiences, and use the same or highly similar processes. There is now quite a body of data lending support to this embodied view of concept representation (e.g., Borghi, Glenberg, \& Kaschak, 2004; Pecher, Zeelenberg, \& Barsalou, 2003, 2004; Zwaan \& Madden, 2005; Zwaan, Stanfield, \& Yaxley, 2002).

In his perceptual symbols theory, Barsalou (1999) provides a detailed account of how cognition is grounded in sensorimotor systems. He proposes that the modality-specific systems that are used for perception and action are also used to represent concepts. During perception and action, the patterns of activation that result from experiences are captured. These form the basis for the perceptual symbols that are used to reenact experiences. During thought a concept is represented by perceptual symbols through a simulation of sensorimotor interaction with the concept. These simulations are partial and sketchy, but nevertheless grounded in embodied experiences. Because embodied experiences involve multiple modalities (vision, audition, touch, taste, smell, motor patterns, and proprioception), symbols used in simulation may also be from any modality of experience. However, selective attention often focuses a simulation on a single modality that is relevant for the current context, rather than simulating all aspects of experience for a given concept.

Pecher et al. (2003) obtained evidence for the selective involvement of different perceptual modalities in conceptual representations. In their study, concept names (e.g., apple) were presented with a property name from a specific modality (e.g., green for visual, or tart for taste) in a property verification task. Pecher et al. found that switching the modality between consecutive trials incurred a processing cost. Responses were faster and more accurate if a target trial (e.g., apple-green) was preceded by a trial from the same modality (e.g., diamond-sparkle) than if it was preceded by a trial from a different modality (e.g., airplane-noisy). Marques (2006) recently replicated these results.

Modality-switch effects in conceptual tasks are explained by assuming that a concept such as apple will be represented mainly by a simulation in the modality that is relevant for a specific context. Thus, if the task is to verify that an apple can be green, a simulation of a visual experience with an apple will be most relevant, whereas if the task is to verify that an apple can be tart, a simulation of a taste experience with an apple will be most relevant. Under these assumptions the modality-switch effect is due to a cost that occurs if between trials attention has to be switched from one modality-specific system to another one. The study of Pecher et al. (2003) was modeled after a study by Spence, Nicholls, and Driver (2001) who obtained a modality-switch effect in perceptual tasks (i.e., detecting the presence of a tone, light flash, or tactile vibration). The finding of an analogous effect in conceptual tasks, such as the property verification task, provides support for the claim that perceptual systems are involved in conceptual representation.

The present study investigated the prediction that if conceptual processing involves running a modality-specific perceptual simulation, conceptual processing should influence performance in a subsequent task that uses nonverbal 
stimuli (i.e., pictures of objects). That is, the effects of Pecher et al. (2003) should generalize to nonlinguistic stimuli. This prediction follows from the perceptual symbols theory (Barsalou, 1999). According to this theory, the processes that are used to represent concepts overlap with those that are used to perceive objects. For example, the processes that are used to represent the color of an apple should overlap with the processes that are used to perceive the color of an apple.

The degree of overlap between representation and perception should also influence memory performance as is predicted by the transfer-appropriate processing principle (Durgunoğlu \& Roediger, 1987; Morris, Bransford, \& Franks, 1977). According to the transfer-appropriate processing principle, performance is affected by the appropriateness of acquisition activities for the kind of memory test. Memory should be better if the same processes are performed on materials during study and test than if different processes are performed. In accordance with the transferappropriate processing principle, a number of studies have shown that recognition memory is better if the presentation format at study overlaps with the presentation format at test. For example, Kazmerski and Friedman (1997) showed that recognition memory for items presented as words at test was better for items studied as words than for items studied as pictures, whereas recognition memory for items presented as pictures at test was better for items studied as pictures than for items studied as words.

In the present study we did not manipulate the presentation format of the concept, but rather the assumed representation of the studied concepts. According to the perceptual symbols theory (Barsalou, 1999), the processes that are used to represent concepts overlap with those that are used to perceive objects. In particular, verifying a visual property involves running a simulation of visual experiences. We tested the hypothesis that memory for pictures of the concepts presented in property verification would be better if a visual property of the concept had been verified than if a nonvisual property of the concept had been verified. For example, verifying that an apple can be green should facilitate subsequent processing of the picture of an apple (i.e., not only the processing of the word apple in a conceptual processing task). This is predicted because the visual simulation involved in verifying that an apple can be green will not only involve the color of apples but also other visual aspects such as the shape of apples.

Evidence that visual representations are activated during language comprehension was obtained by Zwaan and colleagues (2002). They showed that during sentence comprehension performance to pictures is facilitated if they match the situation implied by the sentence (Stanfield \& Zwaan, 2001; Zwaan \& Madden, 2005; Zwaan et al., 2002). In the present study we investigated the modality-specific nature of representations. Rather than looking at performance during online language processing, however, we investigated the role of simulations on a delayed memory task. In a previous study we showed that retrieving knowledge about concepts can be affected by the overlap in modality between study and test (Pecher et al., 2004). That study used only words, however. The present study, therefore, presents a stronger test of the perceptual symbols theory (Barsalou, 1999) because pictures (rather than words) were used in the test task.

In the study phase of Experiment 1 we presented concept names (not pictures) with either a visual property or a property from another modality in a property verification task. In the test phase of the experiment, black-and-white line drawings of the concepts were presented in a recognition memory task. Pictures were presented and subjects decided whether the concept depicted in the picture had been presented earlier in the property verification task. We expected that prior processing of visual properties would facilitate picture recognition memory compared to prior processing of nonvisual properties. Two blocks of property verification and recognition memory tasks were presented to check if strategic use of imagery played a role. If strategic use of imagery during the study phase caused the modality effect subjects would be more likely to use such a strategy during the second study-test block than during the first study-test block. Consequently, if a voluntary imagery strategy was used a larger modality effect would be observed in the second study-test block than in the first study-test block.

\section{Experiment 1}

\section{Method}

\section{Participants}

Thirty-two students at the Erasmus University participated for course credit.

\section{Materials}

A set of 80 familiar concepts from different categories such as animals, fruits, vegetables, and artifacts was created. For each concept (e.g., apple), a visual property (e.g., green), a property from a nonvisual modality (e.g., tart), and a black-and-white line drawing of the concept were selected. The line drawings were taken from Stanfield and Zwaan (2001), Zwaan et al. (2002, http://freud.psy.fsu.edu/ $\sim$ Zwaanlab/studies/index.htm), and Bonin, Peereman, Malardier, Meot, and Chalard (2003, http://leadserv.u-bourgogne.fr/bases/pictures/), or were similar drawings found on the internet. Neither of the two selected properties of a concept was depicted in the line drawing. For the property verification task sentences were created that combined the name of the concept with the name of a property, for example, An apple is green. All properties were correct (i.e., they were true for the concept).

Two counterbalanced versions were created so that each 
concept was combined with a visual property on one list and a nonvisual property on the other list, and each list contained 40 trials in each experimental condition. Half of the concepts in each condition were presented in the first block of the experiment, and the other half were presented in the second block of the experiment. The assignment of trials to blocks was counterbalanced across participants.

In addition, a practice set of 20 sentences and 8 pictures and a filler set of 100 sentences and 80 pictures were created. The practice sentences consisted of 10 correct concept-property sentences and 10 false concept-property sentences. The practice set of eight pictures contained blackand-white drawings of four practiced concepts and four new concepts. The filler sentences consisted of 10 correct sentences and 90 false sentences. The false sentences had properties from various modalities (e.g., visual, taste, auditory) or associated, yet incorrect properties, such as $A$ bed can sleep. ${ }^{1}$ The correct filler sentences were similar to the associated false sentences. The set of filler pictures contained black-and-white drawings of 80 new (unstudied) concepts that served as distractors in the recognition memory task. The filler concepts came from the same categories as the experimental concepts. Each concept was used only once in the experiment, except for the experimental concepts which were presented once as a word in a property verification trial and once as a picture in a recognition memory trial.

\section{Procedure}

The experiment started with written instructions for the property verification task. No mention was made of the recognition memory test that would follow. The property verification task started with a set of 20 practice trials, followed by a block of 40 experimental (all of which were true) and 50 filler ( 5 true and 45 false) trials presented in a random order.

Each property verification trial started with the presentation of a fixation point (*) for $500 \mathrm{~ms}$ on the computer screen. The fixation point was immediately followed by a sentence containing a concept name and a property. The sentence was presented in the center of the screen and remained visible until the participant responded. Participants decided as quickly and accurately as possible whether a property was true of the concept by pressing the $m$ (true) or $\mathrm{z}$ (false) key on the computer keyboard. If the response was incorrect or slower than 2500 ms feedback "Fout" (error) or "Te langzaam" (too slow) was displayed for $600 \mathrm{~ms}$. After the response or feedback a blank screen was presented for $300 \mathrm{~ms}$ before the next trial started.

After the first block of property verification trials written instructions for the recognition memory task were presented. The block of recognition memory pictures started with
4 practice pictures, followed by 40 experimental and 40 filler trials presented in a random order. Half of the trials in each recognition memory block consisted of concepts that had been studied ( 20 with visual properties and 20 with nonvisual properties) and half of the trials consisted of concepts that had not been studied (40 nonstudied fillers). Each trial started with the presentation of a fixation point $(*)$ for $250 \mathrm{~ms}$ on the computer screen. Immediately following the fixation point, the picture was presented in the center of the screen and remained visible until the participant responded. Participants decided whether the concept depicted by the picture had been presented in one of the property verification sentences by pressing the $\mathrm{m}$ (old) or $\mathrm{z}$ (new) key on the computer keyboard. Between trials a blank screen was presented for $250 \mathrm{~ms}$. Feedback on the percentage of correct responses was given at the end of a block of recognition trials. This feedback was given to motivate the subjects and satisfy their curiosity.

After a short break the second block of property verification trials was presented, followed by the second block of recognition memory trials. This second block of memory trials again started with four practice trials to control for recency effects. Presentation of stimuli in the first or second block was counterbalanced.

\section{Results}

The mean reaction times, hit rates (i.e., the percentage of "old" responses to studied concepts) and $d$ ' scores (a measure of sensitivity or memory strength, see MacMillan \& Creelman, 1991) in the recognition memory task are presented in Table 1. Reaction times for incorrect responses and reaction times that were more than two standard deviations from a participant's mean reaction time for correct responses were excluded from the analysis. This resulted in removal of $16.6 \%$ of the data due to errors and $4.1 \%$ of the data due to outlier reaction times.

A two (visual vs. nonvisual property condition) by two (block 1 vs. block 2) repeated measures ANOVA was performed on the mean reaction times. There was a main effect of study modality, in which mean recognition reaction times were faster for concepts that had been studied with a visual property than for concepts that had been studied with a nonvisual property, $F(1,31)=13.51, p<.01$. There was

Table 1. Mean recognition times, hit rates, and $d$ ' scores $(S E)$ in Experiment 1

\begin{tabular}{llll}
\hline Property modality & RT & Hit rate & $d$ \\
\hline Visual & $888(17)$ & $85.4(2.2)$ & $2.45(0.13)$ \\
Nonvisual & $926(20)$ & $81.3(2.2)$ & $2.26(0.12)$ \\
\hline
\end{tabular}

Note. The false alarm rate (i.e., the percentage of "old" responses to nonstudied concepts) was $12.9 \%(S E=1.9)$ and the mean reaction time for correct responses to nonstudied items was $874 \mathrm{~ms}(S E=33)$.

1 We included associated words in the false trials to promote deeper processing (Solomon \& Barsalou, 2004). 
no significant effect of block, $F<1$, nor a significant interaction between block and modality, $F(1,31)=1.00$. If anything, the effect of modality was smaller in the second block (mean modality effect $=26 \mathrm{~ms}$ ) than in the first block (mean modality effect $=53 \mathrm{~ms}$ ) of the experiment.

The ANOVA on hit rates revealed a similar pattern of results. There was a main effect of modality, $F(1,31)=$ $10.92, p<.01$, indicating that the percentage of errors was lower for concepts that had been studied with a visual property than for concepts that had been studied with a nonvisual property. There was no significant block effect, nor a significant interaction between block and modality, both $F$ values $<1$. An ANOVA on the $d$ ' scores confirmed that recognition memory was better for the visual condition than for the nonvisual condition. There was a main effect of modality, $F(1,31)=9.18, p<.01$. There was no significant block effect, nor a significant interaction between block and modality, both $F$ values $<1$.

In addition we analyzed the results of the study phase to determine if there was difference in performance between the visual and nonvisual conditions in the property verification task. Only reaction times that were within two standard deviations of a subject's mean were included in the analysis. The mean trimmed reaction times were $1.071 \mathrm{~ms}$ and $1.060 \mathrm{~ms}$ for trials with visual and nonvisual properties respectively. The mean error percentages were $14.1 \%$ and $13.4 \%$ respectively. These means did not differ significantly between conditions, both $p$ values $>.30$.

\section{Discussion}

The results from the recognition test show that a picture of a concept was recognized faster and with greater accuracy if the name of the concept had been presented earlier in the experiment with a visual property than if it had been presented with a property from a nonvisual modality. This modality effect was not likely the result of participant strategies, because we did not find that the effect increased between blocks.

One interpretation of the present results is that the modality effect is due to more appropriate processing for the picture memory test during study in the visual property condition than during study in the nonvisual property condition. In the visual property condition the perceptual simulation included aspects such as the shape of the concept. Hence, the information stored in memory better matched the picture presented in the recognition memory task than if the concept had been presented in the nonvisual property condition.

Another possible interpretation, however, is that recognition memory was better in the visual property condition than in the nonvisual property condition because presentation of the concept in the visual property condition for some reason resulted in deeper or more elaborate encoding. The analyses of performance in the property verification task suggest that the latter interpretation is not valid because performance did not differ between concepts presented with visual properties and concepts presented with nonvisual properties. However, to further rule out the enhanced encoding explanation of the modality effect we performed a second experiment. In Experiment 2 recognition memory was tested by presenting concept names instead of pictures during test. If the modality effect was due to deeper or more elaborate encoding of the concepts presented with visual properties than for concepts presented with nonvisual properties we should again obtain a modality effect. If, however, the effect that we obtained in Experiment 1 was due to modality-specific representations at study, it should disappear when we tested memory with concept names.

\section{Experiment 2}

\section{Method}

\section{Participants}

Thirty-two students at the Erasmus University participated for course credit.

\section{Materials and Procedure}

The pictures that were used in the recognition memory test of Experiment 1 were replaced by the names of the concepts in the pictures. All other aspects of the present experiment were identical to those of Experiment 1.

\section{Results and Discussion}

Mean reaction times and hit rates in the recognition memory task are presented in Table 2. Reaction times for incorrect responses and reaction times that were more than two standard deviations from a participant's mean reaction time for correct responses were excluded from the analysis. This resulted in removal of $12.8 \%$ of the data due to incorrect responses and $4.6 \%$ of the data due to outlier reaction times. A two (visual vs. nonvisual property condition) by two (block) repeated measures ANOVA was performed on the mean reaction times. There was a significant main effect of block, $F(1,31)=6.20, p<.05$. Reaction times were faster in the first block $(M=746)$ than in the second block $(M=$ 783). The main effect of study modality failed to reach con-

Table 2. Mean recognition times, hit rates, and $d$ ' scores $(S E)$ in Experiment 2

\begin{tabular}{llll}
\hline Property modality & RT & Hit rate & $d^{\prime}$ \\
\hline Visual & $768(17)$ & $88.1(1.7)$ & $2.83(0.09)$ \\
Nonvisual & $757(16)$ & $86.3(1.8)$ & $2.75(0.09)$ \\
\hline
\end{tabular}

Note. The false alarm rate was $7.2 \%(S E=1.0)$ and the mean reaction time for correct responses to nonstudied items was $810 \mathrm{~ms}(S E=21)$. 
ventional levels of significance, $F(1,31)=2.92, p=.10$. If anything, subjects were slower to recognize words that had been presented with a visual property than words that had been presented with a nonvisual property. This result contrasts with the advantage for the visual condition in Experiment 1 . There was no significant interaction between block and modality, $F(1,31)=1.38, p>.10$.

The ANOVA on hit rates showed no main effect of study modality, $F(1,31)=1.48, p>.10$. There was a significant main effect of block, $F(1,31)=11.7, p<.01$, indicating that hit rates were higher in the first block $(M=.88)$ than in the second block $(M=.86)$ of the experiment. There was no interaction between block and modality, $F(1,31)=1.87$, $p>.10$. These findings were confirmed by an ANOVA on the $d$ ' scores. The analysis showed no main effect of study modality, $F(1,31)=1.64, p>.10$. There was a significant main effect of block, $F(1,31)=4.87, p<.05$, indicating that performance was better in the first block (mean $d^{\prime}=$ 2.92) than in the second block of the experiment (mean $d$ ' $=2.67$ ). There was no interaction between block and modality, $F(1,31)=1.18, p>.10$.

In order to see if Experiment 2 had enough power to detect an effect we did a power analysis. The effect sizes in Experiment 1 were .67 for reaction times, .57 for hit rates, and .58 for $d$ 's. The power to find a modality effect was .88 for an effect size of .58 , and .96 for an effect size of .67, both two-tailed (G*Power, Erdfelder, Faul, \& Buchner, 1996). Hence, the absence of a modality effect in Experiment 2 does not appear to be caused by a lack of power.

Somewhat unexpected, performance was better in the first than in the second block of the experiment. One possible explanation for this difference is that during the second block memory retrieval was negatively affected by the stimuli studied in the first block (e.g., Criss \& Shiffrin, 2004). Another explanation might be fatigue. It is not clear, however, why a block effect was present in Experiment 2 but not in Experiment 1. Most important, however, the main effect of block does not affect our main conclusion of Experiment 2, which is that there was no effect of modality when words rather than pictures were used in the test task.

\section{General Discussion}

Recognition memory for pictures was better if the concept name had been presented with a visual property at study than if the concept name had been presented with a nonvisual property at study. The effect was similar in the first and second half of Experiment 1, and disappeared in Experiment 2 when the concept names were used at test instead of the pictures. The absence of an interaction effect between modality and study-test block strongly suggests that the advantage for concepts that had been presented with a visual property was not due to subject strategies. During the first block of property verification trials subjects were unaware that their memory for the concept names would be tested or that pictures of these concepts would be presented. Therefore, it is unlikely that participants used a strategy such as conscious imagery during the first block of property verification trials to enhance their memory. During the second block participants might have used such a strategy, but nevertheless the modality effect did not increase.

One possible explanation for the modality effect observed in Experiment 1 is that visual properties for some reason lead to better encoding at study. For example, the visual properties may have been associated with larger or richer knowledge structures in memory than nonvisual properties, and consequently more elaborate memory traces may have been formed for the concepts presented with visual properties during study. However, if this explanation was correct we should have observed a modality effect in Experiment 2 in which memory was tested with words instead of pictures. The absence of a modality effect in Experiment 2 ruled out this alternative explanation of our results.

We conclude that the modality effect obtained in Experiment 1 is the result of modality-specific processing of concepts at study. Barsalou (1999) has proposed that in order to represent concepts, our sensorimotor systems simulate experiences with those concepts. The involvement of specific sensorimotor systems is context dependent. During our property verification task participants simulated experiences with the concepts in order to verify the properties. When a visual property was presented, such as green in the sentence An apple is green, they would simulate a visual experience with an apple. During that simulation other visual properties of apple such as its shape were also represented. When a nonvisual property such as tart was presented, however, the visual system was not (or less) involved in running the simulation. Therefore, visual properties of apple were not included (or to a lesser extent) in the representation of apple. When memory was subsequently tested with a black-and-white picture of an apple there was a better match between the test stimulus and the representation stored in memory for the visual property condition than for the nonvisual property condition.

The present results provide an important extension to those of Pecher et al. (2003). In their study, a modalityswitch effect was found between trials in a property verification task, analogous to the modality-switch effect that can be found in perceptual tasks (Spence et al., 2001). Pecher et al. concluded that the conceptual system is grounded in sensorimotor systems. In their study, however, only conceptual trials consisting of verbal materials (i.e., concept-property sentences) were presented. Moreover, the modality-switch effect that they obtained was the result of the trial immediately preceding the target trial. Thus, they showed that sensorimotor information played a role during online sentence processing. In the present study we tested whether verifying properties of concepts resulted in the storage of perceptual information in memory. The finding that recognition memory for pictures was better if the con- 
cepts had been presented with visual properties during study supports the idea that conceptual processing involves perceptual simulation. In addition, it shows that a modality-specific representation was stored in memory.

Additional evidence for sensorimotor involvement comes, for example, from studies in which participants read or hear language, followed by the presentation of visual information that matches or mismatches the perceptual experience that is implied but not actually described by the language. For example, Zwaan et al. (2002) presented sentences such as The eagle is in the sky or The eagle is in its $n e s t$. In the first case the actual visual experience would be of an eagle with its wings stretched out, and in the second case it would be of an eagle with its wings folded in. A picture of an eagle immediately followed the sentence, and processing of the picture was facilitated if it matched the shape implied by the preceding sentence. Other studies have obtained similar effects for implied orientation (Richardson, Spivey, Barsalou, \& McRae, 2003; Stanfield \& Zwaan, 2001), movement (Zwaan \& Madden, 2005), and action (Borghi, 2005; Borghi et al., 2004; Glenberg \& Kaschak, 2002). This type of evidence is consistent with theories that assume sensorimotor simulation of language materials and would not be predicted by amodal theories (Stanfield \& Zwaan, 2001).

In the present study we have extended these findings. We have manipulated which modalities were involved in conceptual representation. Our results show that this may vary according to the context. In addition, our results show that the effect of modalities has long-lasting effects on memory. In previous studies the perceptual stimulus immediately followed the sentence in which the concept was named. These studies showed that sensorimotor information was activated during online sentence processing. Zwaan et al.'s results (and those of similar studies) show that such representations match actual perceptual processes or actions. The results from Pecher et al. (2003) show that attention may focus selectively on a single modality. The present study shows that sentence processing results in the storage of a modality-specific representation. The finding that these sensorimotor effects of language processing remain for longer time is new and of potential importance.

An even more convincing result might have been to find better performance in a different modality condition for stimuli in that modality, for example, sound or touch. Unfortunately, this is impossible with the present experimental design. In Experiment 1 the visual properties that were used in the property verification task were not perceivable in the perceptual stimuli. For example, many of the properties were color names, whereas black and white drawings were used during the memory test. Thus, the information overlap between study and test was the same in de visual and the nonvisual condition. For other modalities, however, most concepts do not have multiple properties that can be separated perceptually. Hence, if we wanted to test other modalities we would need a different design.

A number of published studies have shown that various memory measures are sensitive to the meaning context at study for words (e.g., Anderson, Pichert, Goetz, Schallert, Stevens, \& Trollip, 1976; Barclay, Bransford, Franks, McCarrell, \& Nitsch, 1974; Barsalou 1993; Zeelenberg, Pecher, Shiffrin, \& Raaijmakers, 2003). These studies did not look at sensorimotor information, and used only words as stimuli. Nevertheless, the context effects in these studies may have been due to the involvement of different modalities in the different context conditions used in the experiments. The present results provide a possible mechanism in the form of sensorimotor simulations for these previous findings.

Thus, the present results provide further support for the view that sensorimotor systems are involved in language comprehension. As people are giving meaning to language materials, they form mental representations using perceptual symbols (Barsalou, 1999). The present study shows that these representations are simulations that match actual perceptions, providing evidence that the same systems are used for perception and conceptual representation. The present study also shows that earlier representations can affect later processing of the same concepts.

\section{Acknowledgments}

Kiki Zanolie is now at Leiden University, The Netherlands. This research was supported by a grant from the Netherlands Organization for Scientific Research (NWO) to Diane Pecher. We would like to thank Carol Madden and Saskia van Dantzig for helpful suggestions on earlier versions of this manuscript.

\section{References}

Anderson, R.C., Pichert, J.W., Goetz, E.T., Schallert, D.L., Stevens, K.V., \& Trollip, S.R. (1976). Instantiation of general terms. Journal of Verbal Learning and Verbal Behavior, 15, 667-679.

Borghi, A.M., Glenberg, A.M., \& Kaschak, M.P. (2004). Putting words in perspective. Memory and Cognition, 32, 863-873.

Barclay, J.R., Bransford, J.D., Franks, J.J., McCarrell, N.S., \& Nitsch, K. (1974). Comprehension and semantic flexibility. Journal of Verbal Learning and Verbal Behavior, 13, 471-481.

Barsalou, L.W. (1993). Flexibility, structure, and linguistic vagary in concepts: Manifestations of a compositional system of perceptual symbols. In A.F. Collins, S.E. Gathercole, M.A. Conway, \& P.E. Morris (Eds.) Theories of memory (pp.30-39). Hove, UK: Erlbaum.

Barsalou, L.W. (1999). Perceptual symbol systems. Behavioral and Brain Sciences, 22, 577-660.

Bonin, P., Peereman, R., Malardier, N., Meot, A., \& Chalard, M. (2003). A new set of 299 pictures for psycholinguistic studies: French norms for name agreement, image agreement, conceptual familiarity, visual complexity, image variability, age of ac- 
quisition, and naming latencies. Behavior Research Methods, Instruments, and Computers, 35, 158-167.

Borghi, A.M. (2005). Object concepts and action. In D. Pecher \& R.A. Zwaan (Eds.), The grounding of cognition: The role of perception and action in memory, language, and thinking (pp. 8-34). Cambridge: Cambridge University Press.

Borghi, A.M., Glenberg, A.M., \& Kaschak, M.P. (2004). Putting words in perspective. Memory and Cognition, 32, 863-873

Criss, A.H., \& Shiffrin, R.M. (2004). Context noise and item noise jointly determine recognition memory: A Comment on Dennis and Humphreys (2001). Psychological Review, 111, 800-807.

Durgunoğlu, A.Y., \& Roediger, H.L. (1987). Test differences in accessing bilingual memory. Journal of Memory and Language, 26, 377-391.

Erdfelder, E., Faul, F., \& Buchner, A. (1996). GPOWER: A general power analysis program. Behavior Research Methods, Instruments, and Computers, 28, 1-11.

Glenberg, A.M. (1997). What memory is for. Behavioral and Brain Sciences, 20, 1-55.

Glenberg, A.M., \& Kaschak, M.P. (2002). Grounding language in action. Psychonomic Bulletin and Review, 9, 558-565.

Goldstone, R.L., \& Barsalou, L.W. (1998). Reuniting perception and conception. Cognition, 65, 231-262.

Kazmerski, V.A., \& Friedman, D. (1997). Old/new differences in direct and indirect memory tests using pictures and words in within- and cross-form conditions: Event-related potential and behavioral measures. Cognitive Brain Research, 5, 255-272.

MacMillan, N.A., \& Creelman, C.D. (1991). Detection theory: A user's guide. Cambridge: Cambridge University Press.

Marques, J.F. (2006). Specialization and semantic organization: Evidence for multiple-semantics linked to sensory modalities. Memory and Cognition, 34, 60-67.

Morris, D.C., Bransford, J.D., \& Franks, J.J. (1977). Levels of processing versus transfer appropriate processing. Journal of Verbal Learning and Verbal Behavior, 16, 519-533.

Pecher, D., Zeelenberg, R., \& Barsalou, L.W. (2003). Verifying conceptual properties in different modalities produces switching costs. Psychological Science, 14, 119-124.

Pecher, D., Zeelenberg, R., \& Barsalou, L.W. (2004). Sensorimotor simulations underlie conceptual representations: Modalityspecific effects of prior activation. Psychonomic Bulletin and Review, 11, 164-167.

Pulvermüller, F. (1999). Words in the brain's language. Behavioral and Brain Sciences, 22, 253-336.
Richardson, D.C., Spivey, M.J., Barsalou, L.W., \& McRae, K. (2003). Spatial representations activated during real-time comprehension of verbs. Cognitive Science, 27, 767-780.

Spence, C., Nicholls, M.R., \& Driver, J. (2001). The cost of expecting events in the wrong sensory modality. Perception and Psychophysics, 63, 330-336.

Stanfield, R.A., \& Zwaan, R.A. (2001). The effect of implied orientation derived from verbal context on picture recognition. Psychological Science, 12, 153-156.

Wilson, M. (2002). Six views of embodied cognition. Psychonomic Bulletin and Review, 9, 625-636.

Zeelenberg, R., Pecher, D., Shiffrin, R.M., \& Raaijmakers, J.G.W. (2003). Semantic context effects and priming in word association. Psychonomic Bulletin and Review, 10, 653-660.

Zwaan, R.A. (2004). The immersed experiencer: Toward an embodied theory of language comprehension. In B.H. Ross (Ed.), The psychology of learning and motivation (Vol.43, pp. 35-62). New York: Academic Press.

Zwaan, R.A., \& Madden, C.J. (2005). Embodied sentence comprehension. In D. Pecher \& R.A. Zwaan (Eds.), The grounding of cognition: The role of perception and action in memory, language, and thinking. Cambridge: Cambridge University Press.

Zwaan, R.A., Stanfield, R.A., \& Yaxley, R.H. (2002). Language comprehenders mentally represent the shapes of objects. Psychological Science, 13, 168-171.

Received March 6, 2006

Revision received June 1, 2006

Accepted June 1, 2006

Diane Pecher

Psychology Department, T12-33

Erasmus University Rotterdam

Postbus 1738

NL-3000 DR Rotterdam

The Netherlands

Tel. +3110408 8625

Fax +31 104089009

E-mail pecher@fsw.eur.nl 